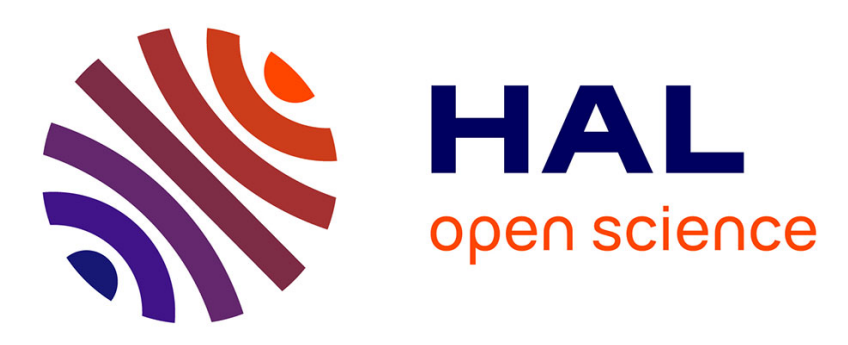

\title{
Subjective and objective evaluations of feature selected multi output filter for speckle reduction on ultrasound images
}

M Outtas, Lu Zhang, O Deforges, A Serir, Wassim Hamidouche, Y Chen

\section{- To cite this version:}

M Outtas, Lu Zhang, O Deforges, A Serir, Wassim Hamidouche, et al.. Subjective and objective evaluations of feature selected multi output filter for speckle reduction on ultrasound images. Physics in Medicine and Biology, 2018, 63 (18), pp.185014. 10.1088/1361-6560/aadbc9 . hal-01880192

HAL Id: hal-01880192

https://hal-univ-rennes1.archives-ouvertes.fr/hal-01880192

Submitted on 14 Dec 2018

HAL is a multi-disciplinary open access archive for the deposit and dissemination of scientific research documents, whether they are published or not. The documents may come from teaching and research institutions in France or abroad, or from public or private research centers.
L'archive ouverte pluridisciplinaire HAL, est destinée au dépôt et à la diffusion de documents scientifiques de niveau recherche, publiés ou non, émanant des établissements d'enseignement et de recherche français ou étrangers, des laboratoires publics ou privés. 


\title{
Subjective and Objective Evaluations of feature selected Multi Output Filter for Speckle reduction on Ultrasound Images
}

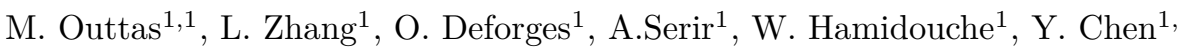 \\ ${ }^{d}$ LTIR, University of Sciences and Technology Houari Boumedienee, Algiers, Algeria \\ ${ }^{e}$ VAADER-IETR National Institute of Applied Sciences (INSA) Rennes, France \\ ${ }^{f}$ Laboratory of Image Science and Technology, Southeast University, Nanjing 210096, China
}

\begin{abstract}
Ultrasonographic examination, either as visual inspection or quantitative analysis, is the most widely diagnostic resource. However, speckle noise is one of the drawbacks that makes it less effective than other medical imaging systems. Several speckle reduction methods often offer effective speckle reduction but generally suffer from oversmoothing, a blurring effect and a man-made appearance. In this paper, we propose a Multi-Output Filter based on the Multiplicative Multiresolution Decomposition (MOF-MMD). This multi-scale based method enables the enhancement of the original image and provides three enhanced outputs: global, edge and texture images. The multi-output filter aims at offering an enhanced image according to the features desired by radiologists. The different structures, textures and edges are filtered according to the contour image obtained by morphological operators. Three radiologists with different years of experience, have subjectively evaluated the speckle reduction methods according to enhanced features. The results of objective metrics and subjective evaluation showed that the proposed method reduces speckle and could help radiologists, according to their years of experience, in the diagnostic task. Finally, the correlation between three objective metrics and the perceived quality of contrast, diagnostic, texture and edges show that an objective metric is suitable for assessing quality of ultrasound images.
\end{abstract}

Keywords: Subjective evaluation, Objective metrics, Ultrasound, Medical images, Speckle, Multi-Output filter, Multiplicative Multiresolution Decomposition (MMD) .

\section{Introduction}

The great potential of Ultrasound (US) imaging in diagnostics means that it is viewed as the stethoscope of the future. Ultrasonographic examination is

\footnotetext{
* Corresponding author
} 
carried out in two ways: qualitative visual inspection based on the clinician's interpretation and quantitative analysis by extracting measures or biomarkers that aid in the diagnosis. In obstetrics, measurement of the nuchal translucency can detect a number of anomalies such as trisomy or congenital heart disease. Moreover, the measurement of nuchal fold thickness is a marker for Down syndrome with a false positive rate of $1.4 \%$ ?

In Gastroenterology, liver parenchymal texture is a subjective characteristic for the detection of cirrhosis ?. Moreover, the Sonographic HepatoRenal Index (SHRI) is a measure based on comparison between liver and kidney brightness. It has been reported that more than 34 biopsies could have been avoided among a series of 101 performed for different diagnostic reasons if the SHRI method had been used prospectively?.

Nevertheless, US imaging has a main drawback of visual quality. It shows a granular structure called "speckle", making visual interpretation difficult. Speckle is an undesirable property of the image as it masks small differences in gray level ? ?. This specific artefact for US, corrupts the image in a multiplicative manner. The speckle in a clinical image is generated mainly by constructive and destructive interference of subresolution tissue scatterers at fixed spatial locations. It appears as a light and dark mottled grainy pattern.

Speckle is detrimental, because it adds texture to the image making it more difficult to discern subtle variation in image signal, particularly for small structures and boundaries.

For instance, on a conventional B-mode US image of a liver, the speckle pattern changes with both steatosis and fibrosis. This can make the changes in microarchitecture imperceptible to the naked eye and lead to misinterpretation of the US image ? ?. Finally, speckle impedes post-processing techniques such as image segmentation, registration, data classification, texture analysis or even automatic diagnostic application.

However, this "granular" texture (speckle) is not truly noise in the typical engineering sense because its texture often carries useful information about the image being viewed ?. Many researchers have investigated the statistical specificities of speckle in ultrasound images since the seventies such as ? and et al. ?. In this last study about statistics of speckle in ultrasound, the authors point out that the texture in the image of parenchymal tissue could be viewed either as image signal or undesirable noise ?. Hence, it is necessary to reduce the speckle while preserving meaningful data.

A large amount of research has investigated speckle reduction and improved the quality of US images. There are two main categories of despeckling methods: compounding methods and post-processing ones. The compounding speckle reduction methods include both spatial and frequency compounding ?. Postprocessing speckle reduction techniques are widely used. These techniques decrease speckle after the US image is formed.

However, speckle reduction techniques present some limitations such as oversmoothing of texture, loss of subtle details during the filtering process or moreover blurring the edges. Finally, some filtering methods give an artificial appearance to the enhanced images?. 
We can notice that the existing speckle reduction studies propose only a unique viewing possibility, while the interpretation of US image is based on multiple evaluation tasks: general aspect, echo pattern, outer border and size.

To counter this problem, we extend our recently proposed Multi-Output Filter based on Multiplicative Multiresolution Decomposition (MOF-MMD) ?. In this paper we propose to make the MOF-MMD where the enhancement of different characteristics of the image implies the calculation of noise statistics performed automatically with the respect of extracted features and multi-resolution analysis to make this algorithm more practical in daily clinical practice.

Since the end-user of the ultrasound images is the radiologist, we believe that it is more reasonable to evaluate the algorithm using human observers. Thus, the second part consists in conducting a subjective experiment involving three radiologists with different years of experience to assess the final perceived quality of filtered in vivo abdominal liver US images. The correlations between the subjective scores and the objective metrics' outputs will be presented and analyzed. The impact of the radiologists' years of experience on their way of scoring will also be discussed. The MOF-MMD filter is compared with two recent and efficient speckle reduction filters: Optimized Bayesian NL-Means with block selection (OBNLM) ? and Anisotropic Diffusion filter with Memory based on Speckle Statistics (ADMSS) ?.

The rest of this paper is structured as follows. In section ?? related works are presented. Section ?? describes the proposed fully automatic multi-output filter. In Section ?? the dataset, objective metrics and subjective tests are depicted. Section ?? discusses and analyzes the experimental results. Finally, Section ?? concludes the paper with some perspectives.

\section{Related works}

As described in Section ?? many filters were developed to attempt to reduce speckle in US images. Within the state-of-the-art, image speckle reduction approaches could be categorized into four types namely, adaptive statistics, anisotropic diffusion, multi-scale analysis and non-local based filters.

\subsection{Adaptive local statistics filters}

The adaptive filters are based on the statistical information calculated from a pre-defined neighborhood. Indeed, multiplicative speckle noise corrupts the Synthetic Aperture Radar (SAR) images in the same manner as it does US images. These filters were initially proposed to reduce speckle in SAR data. The Median filter ? replaces the gray level of the neighborhood's center pixel with the median graylevel of the considered neighborhood. The statistical properties of the image were used by Lee filter ? ?, Frost ? and Kuan ? who proposed to reduce speckle to obtain a speckle-free image by the Minimum Mean Square Error (MMSE) criterion. 


\subsection{Anisotropic diffusion filter}

The Anisotropic Diffusion (AD) process was introduced by Perona and Malik ? for smoothing images. Yu and Acton ?, by introducing Speckle Reducing Anisotropic Diffusion filter (SRAD), applied anisotropic diffusion filter for the first time to speckle noise. This was done by introducing the instantaneous coefficient of variation for an edge-sensitive speckle reduction. An improved version of the SRAD was then proposed with the Detail Preserving Anisotropic Diffusion (DPAD) ?. This improved version of the SRAD includes an estimation of the variation coefficient of both signal and noise based on the Kuan filter. In ? the authors extended the SRAD and DPAD methods to an Oriented Speckle Reducing Anisotropic Diffusion (OSRAD). This method is combined with matrix anisotropic diffusion allowing different levels of filtering in the gradient and the principal curvature directions. In ?, an Anisotropic Diffusion filter with Memory based on Speckle Statistics (ADMSS) was proposed. This recent method embeds a memory mechanism that speeds up the diffusion process in meaningless regions and adaptively preserves relevant structures.

\subsection{Multi-scale filter}

Several multi-scale based methods were proposed for speckle reduction in ultrasound imaging mainly based on wavelet, curvelet or contourlet transforms. Most of these filters use the wavelet transform, as the wavelet theory provides a powerful representation of the image and is widely used for image processing such as image compression, segmentation and noise reduction.

The wavelet-based speckle reduction filters include three main steps: wavelet decomposition, modification of wavelet coefficients, reconstruction of the modified wavelet coefficients and "noise free coefficient" by invert wavelet transform. The wavelet shrinkage denoising was first proposed by ?, later this thresholding method was applied to medical imaging for speckle reduction ?. The authors in ? proposed to balance the degree of noise reduction for the preservation of relevant details. Extension of wavelet transform are also involved in despeckling: dual tree complex wavelet transform in ? or monogenic wavelet transform ?. Other multi-scale approaches based on the pyramid transform were suggested to reduce speckle in US images as in ???

\subsection{Non-local means filter}

The Non-Local (NL) approach is a new paradigm that proposes to replace the local comparison of pixels by the non-local comparison of patches ?. Firstly introduced in ?, NL-means methods were used for US noise reduction with a Bayesian formulation by Coupé et al. ? in the Optimized Bayesian NL-means (OBNLM) filter. The OBNLM introduces a Pearson distance to compare nonlocal patches and select the most relevant, and uses it as features for denoising images. Related hereto, ? propose to incorporate a Gamma model in the NLmeans denoising. In ? and ?, Yang et al. found that increasing the searching region can lead to improved noise-suppression performance in low dose CT image processing. In ?, they incorporated a structure-adaptive fuzzy estimation into 
iterative NL-means for random-valued noise estimation. It was also found that NL-means estimation can be used to build the regularization term for medical image reconstruction ?. The main drawbacks of these methods are the oversmoothing of the images, and the computational complexity.

The review of the state-of-the art methods shows that: first, the speckle reduction methods either oversmooth the ultrasound or do not reduce speckle significantly in some regions; second, all the algorithms provide only one viewing possibility, while doctors may have different requirements of image features in different situations. These motivate us to propose an improved speckle filtering method without oversmoothing effect and with multiple viewing possibilities.

\section{Proposed method (MOF-MMD)}

The proposed MOF-MMD is schematically represented in Fig. ??. It consists of five steps:

- Preliminary step of features-like segmentation achieved using morphological operators;

- Calculation of noise level on local window;

- Multi-scale decomposition using MMD;

- Thresholding process according to the features;

- Reconstruction of enhanced images using MMD synthesis.

The details of each step are given below.

\subsection{Multiplicative multiresolution decomposition}

\subsubsection{Multiplicative decomposition}

The nonlinear multiplicative decomposition is a multi-scale analysis/synthesis representation of $2 \mathrm{D}$ signals ?. This nonlinear decomposition is suitable for multiplicative noise reduction and has been used to reduce multiplicative noise in Synthetic Aperture Radar? and medical US images ?. It uses filter banks with critical sub-sampling and perfect reconstruction (reversible). In ? the authors consider a description of the analysis and the synthesis inputs-outputs systems with equal symbol rates at both the input and the output. The image is decomposed into an approximate output subband $y_{1}$ and three detail images $y_{2 h}$, $y_{2 v}$ and $y_{2 d}$ characterized by horizontal, vertical and diagonal directions. The desired structure is obtained by performing a polyphase decomposition of the 2D signal (the image) ?. To avoid division by zero, the value 1 is added to the original image to provide the input image $(I)$. The four poly-phase components $x_{11} ; x_{12} ; x_{21}$ and $x_{22}$ of the input image $I$ of size $N \times M$ are defined by:

$$
x_{i j}(n, m)=I(2(n-1)+i, 2(m-1)+j) \quad i, j \in\{1,2\}
$$

where $n=1, \ldots, \frac{N}{2}$ and $m=1, \ldots, \frac{M}{2}$ 


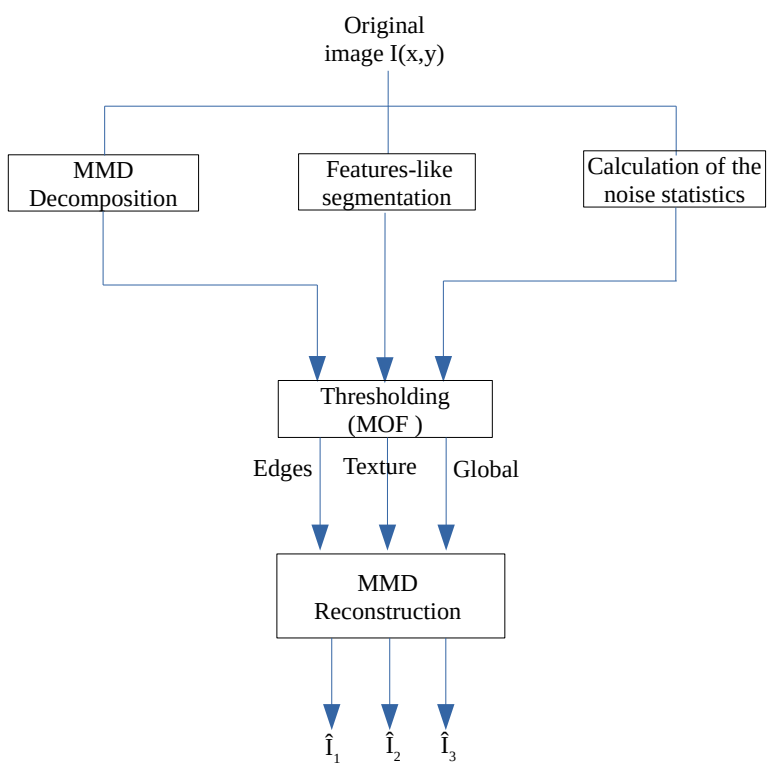

Figure 1: MOF-MMD Diagram

The multiplicative decomposition could be used within its undecimated version, with an equal number of coefficients at each resolution scale. The four polyphase components for practical implementation of this undecimated algorithm are defined by:

$$
x_{i j}(n, m)=I(n+i-1, m+j-1) \quad i, j \in\{1,2\}
$$

where $\mathrm{n}=1, \ldots, \mathrm{N}$ and $\mathrm{m}=1, \ldots, \mathrm{M}$

For $(i, j) \in\{1,2\}$, the linear filter $h_{i j}$ and $f_{i j}$ are given by:

$$
\left\{\begin{array}{r}
h_{i j}(k, l)=h((2 k+1)+i, 2(l+1)+j) \\
f_{i j}(k, l)=\frac{1}{h_{i j}(k, l)}
\end{array}\right.
$$

where $h$ and $f$ are bi-dimensional linear filters. The approximation $y_{1}$ is given by

$$
y_{1}=\sum_{i=1}^{2} \sum_{j=1}^{2} h_{i j} x_{i j} .
$$

The nonlinear analysis filters $D$ illustrated in Fig. ??, is defined by the following equations:

$$
y_{2 v}=\left\{\begin{array}{cc}
\beta \frac{x_{12}}{x_{11}}, & x_{11} \geq x_{12} \\
\beta\left(2-\frac{x_{11}}{x_{12}}\right) & \text { otherwise }
\end{array}\right.
$$




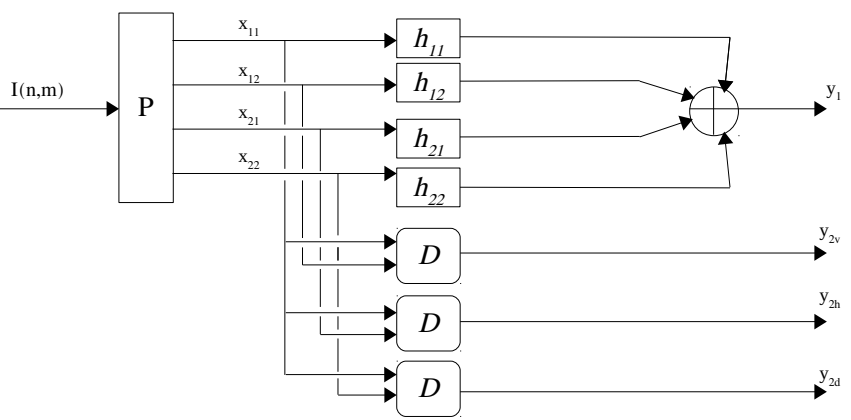

Figure 2: The 2D MMD analysis scheme

$$
\begin{aligned}
& y_{2 h}=\left\{\begin{array}{cc}
\beta \frac{x_{21}}{x_{11}}, & x_{11} \geq x_{21} \\
\beta\left(2-\frac{x_{11}}{x_{21}}\right) & \text { otherwise }
\end{array}\right. \\
& y_{2 d}=\left\{\begin{array}{cc}
\beta \frac{x_{22}}{x_{11}}, & x_{11} \geq x_{22} \\
\beta\left(2-\frac{x_{11}}{x_{22}}\right) & \text { otherwise }
\end{array}\right.
\end{aligned}
$$

where $\beta$ is a positive scalar fixed to 0.5 . The details $y_{2 h}, y_{2 v}$ and $y_{2 d}$ vary within the interval $[0 ; 1]$. It should be noted that high contrasted details correspond to values far from $\beta$, whereas, values close to $\beta$ correspond to smooth regions.

\subsubsection{Multi-resolution decomposition}

Multi-resolution decomposition is based on subband decomposition using analysis filter bank that operates at different stages of the outputs. The approximate output subband $y_{1}$ (the low-frequency coefficients) is decomposed into one or more coefficient outputs of the preceding stage. In the subband, $y_{1}$ is split into its polyphase $y_{11}, y_{12}, y_{21}$ and $y_{22}$ and then filtered. At the first resolution $j=1$ :

$y_{11}^{(j)}=x_{11}, y_{12}^{(j)}=x_{12}, y_{21}^{(j)}=x_{21}$ and $y_{22}^{(j)}=x_{22}$

At the highest resolution $J$, the original discrete image is represented by the set $R$ defined by

$$
R=\left(y_{1}{ }^{(j)},\left(y_{2 h}{ }^{(j)}, y_{2 v}{ }^{(j)}, y_{2 d}{ }^{(j)}\right)\right)_{2 \leq j \leq J}
$$

Fig. ?? illustrates the undecimated multiplicative multi-resolution decomposition for one resolution level of an US image of an agar gel phantom that mimicking the phenomena of the inhomogeneous scattering. This phantom was homemade using: $12 \mathrm{~g}$ gelatin, 8 grs agar agar, $500 \mathrm{ml}$ hot water, $40 \mathrm{ml}$ glycerin. A latex glove finger filled with water was immersed to mimic a cyst. The upper left panel is the approximation image of the original image constituted by the low-frequency coefficients. The bright ring is the Cyst mimic. 


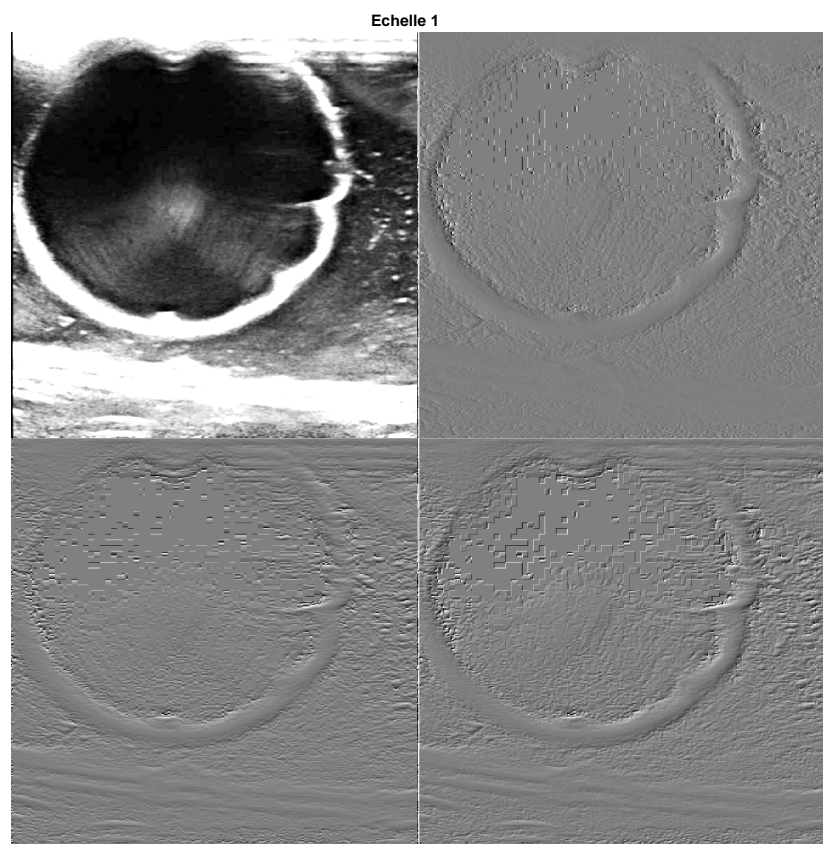

Figure 3: 2D MMD undecimated decomposition of US image of an agar gel phantom: approximation image (upper left), detail images vertical, horizontal and diagonal

\subsection{Features-like segmentation by morphological operator}

In this paper, the features-like segmentation enables the enhancement of the different features and structures of the image. It is performed using mathematical morphology.

\subsubsection{Morphological Operators}

Morphological operators are used here to segment features-like structures. Indeed, mathematical morphology is a well established domain used for image analysis. Based on the algebra of non-linear operators, it performs better and faster than the standard approaches in many tasks such as pre-processing, segmentation using object shape and object quantification ?. We will define some basic morphological operators. Firstly, a shape parameter called structuring element, characterized by its shape and size, is used to perform morphological techniques. Depending on the type of morphological transformation, the pixel value is set to the minimal or maximal value of the pixels?.

\subsubsection{Features-like segmentation}

As medical images generally contain more round shapes than straight lines and angles, the disk-shaped structuring element is a more appropriate choice. An algorithm for the efficient computation of morphological operations for gray images with a circular structuring element has been proposed in?. 
Let $I$ be the original US image and $C$ the circular structuring element with a radius of eight pixels. The features-like segmentation is obtained as follows:

$$
\begin{gathered}
I_{(o)}=I \circ C \\
I_{(o c)}=I_{(o)} \bullet C \\
I_{(e)}=I_{(o c)} \ominus C \\
I_{(d)}=I_{(o c)} \oplus C \\
S=I_{(d)}-I_{(e)}
\end{gathered}
$$

where $\oplus, \ominus, \circ$ and $\bullet$ denote dilatation, erosion, opening and closing operators, respectively. $S$ represents the contour image obtained by morphological treatment and illustrated in Fig. ??.

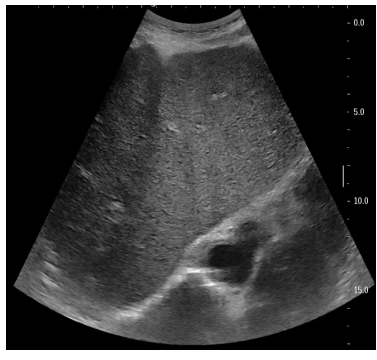

(a) Original image

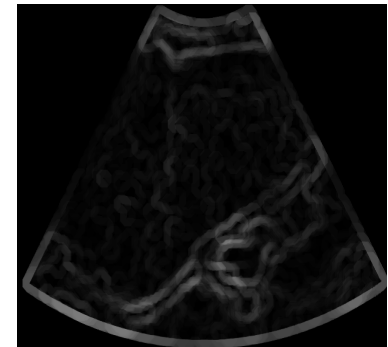

(b) Contour image

Figure 4: Features-like segmentation

\subsection{Calculation of Noise Statistics}

The MOF-MMD is sensitive to noise statistics, indeed the thresholding step depends on the knowledge of these statistics. Therefore, we propose an automatic method to select a homogeneous window $H_{\text {area }}$ to calculate the noise. The speckle filtering framework proposes an automatic estimation of the noise variance ?. In the case of homogeneous areas $H_{\text {area }}$, where the signal component can be considered constant and the image variation is only attributable to noise, the coefficient of variation $R$ of the speckle noise is given by:

$$
R=\frac{S t d_{H_{\text {area }}}}{\mu_{H_{\text {area }}}}
$$

where $S t d_{H_{\text {area }}}$ and $\mu_{H_{\text {area }}}$ stand for standard deviation and mean values of $H_{\text {area }}$, respectively. The location of $H_{\text {area }}$ require that: 


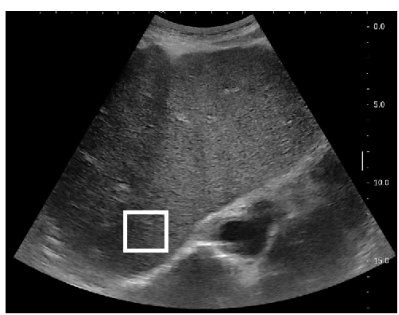

(a) Auomatic selection

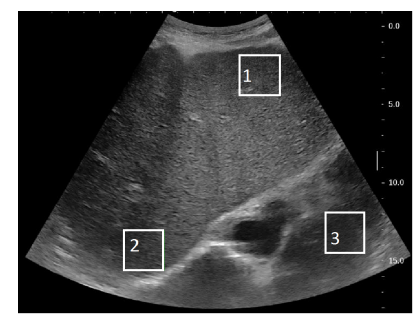

(b) Manual selection

Figure 5: Local window selection for calculation of $C_{n}$ and $T$

- The $H_{\text {area }}$ must be inside the extracted features features (contour $S$ ) obtained in the previous Section ??.

- The $H_{\text {area }}$ has to be homogeneous area. In order to detect $H_{\text {area }}$ we use MMD's coefficient properties (described in Section ??), in smooth regions, the values of the MMD's coefficient components are close to $\beta$. $H_{\text {area }}$ corresponds to the one that minimized the median absolute deviation mad around $\beta$

$$
\operatorname{mad}=\frac{1}{n} \sum_{i=1}^{n}\left|y_{2(h, v, d)}^{1}(i)-\beta\right|_{i \in H_{\text {area }}}
$$

The noise level is then calculated within the selected window $W=H_{\text {area }}$. So, at each resolution, one could estimate the normalized standard deviation

$$
C_{n}=\sqrt{\frac{\operatorname{var}_{W}}{\mu_{W}}}
$$

where $\operatorname{var}_{W}$ the variance and $\mu_{W}$ the mean value of the selected window. The latter parameter is defined in this section and it will be used later in this paper as a parameter of our method for speckle reduction.

The manual selection of multiple windows $W$ is possible, thanks to the quality criterion selection (cf. Section ??). Fig. ?? and Fig. ?? depict the results of obtained areas by using automatic and manual processes, respectively. A good agreement is obtained by automatic calculation of noise statistics. rred This automation makes it possible to divide the length of the manual selection process by at least $\mathrm{N}$ (number of manually selected windows).

\subsection{Thresholding: Multi-Output filter}

The existing speckle reduction methods propose a unique enhancement for the overall image. Sometimes a stopping criterion is applied for specific structures of the image. Nevertheless, there is only one viewing possibility. Herein, we propose a new method that allows to the medical observer to view multiple enhancement possibilities of the original US image. This can respond to the 
specific need for a diagnosis, according to which it is more relevant to examine edges, texture, thickness, etc.

As indicated in Section ??, in smooth regions the values of the MMD's coefficient components are close to $\beta$. This property helps to enhance the image while preserving structural details and avoiding a blurring effect. In the rest of this section, we will refer to MMD coefficient details $y_{2 H}, y_{2 V}, y_{2 D}$ by $D_{C}$ where $C$ stands for ' $H^{\prime}$ horizontal, ' $V$ ' vertical and ' $D$ 'diagonal details.

The selection of the optimal threshold is the main limitation of the thresholding filter. To overcome this we propose to adapt thresholding to the local context. Thus, this is done according to the pixel intensity of the images obtained from features-like segmentation. This allows multiple enhancement possibilities of the original US image.

In this aim, at each resolution $j$, the MMD's coefficient $D_{C}^{(j)}$ is thresholded in three ways to provide the thresholded coefficient $\hat{D}_{C, k}^{(j)}$ with $k=1,2,3$.

\subsubsection{Filter first output - Sharp edge enhancement}

This first output of the filter is an image with enhanced edges. It is pertinent for measures conducted in US images such as lesion size, distance and so on. For each pixel of the contour image, a thresholding is applied as follows:

$$
\hat{D}_{C, 1}^{(j)}=\left\{\begin{array}{l}
\beta \quad \text { if } \quad \beta-\beta_{t} \leq D_{C}^{(j)} \leq \beta+\beta_{t} \\
D_{C}^{(j)} \times \nu-(\gamma \times \alpha \times S) \quad \text { if } \quad D_{C}^{(j)}+\tau \leq \beta \\
D_{C}^{(j)} \times \nu+(\gamma \times \alpha \times S) \quad \text { if } \quad D_{C}^{(j)}-\tau \geq \beta \\
\beta \quad \text { otherwise }
\end{array}\right.
$$

where $D_{C}^{(j)}$ and $\hat{D}_{C, 1}^{(j)}$ represent the MMD's coefficients at scale $j$ of the original noisy image $I$ and its thresholded version, respectively. $\beta_{t}$ is a threshold that aims to reduce the speckle of the coefficient components in the smoothest pixels. $\beta_{t}$ is set experimentally to 0.0016. $\tau=(T \times \nu \times \alpha \times S)$, with $T=C_{n} \times j / J$ is the threshold calculated from the noise level at each scale $j$ while $J$ represents the number of scales. $\alpha$ is set to 0.25 to avoid the displacement of edge pixels in the filtered images. $\nu$ and $\gamma$ are given by $\nu=\frac{1}{\sqrt{1+C_{n}^{2}}}$ and $\gamma=1-\frac{1}{\sqrt{1+C_{n}^{2}}}$. Notice that the thresholding is proportional to the contour images i.e., the thicker is the edges the more it is enhanced.

\subsubsection{Filter second output - Texture enhancement}

This second output of the filter is an image with enhanced texture. It is pertinent to enhance texture by reducing speckle while preserving the texture pattern. Let $\bar{S}$ denote the image of pixels that belong to the original image and do not belong to the contour $S$. The second output results from the following 
thresholding:

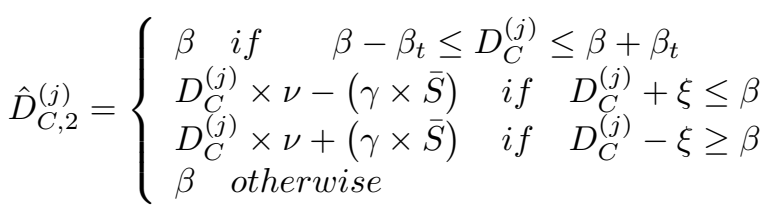

with $\xi=T \times \nu \times \bar{S} t$,

\subsubsection{Filter third output - Global image enhancement}

The third output of the filter is a global enhancement of the image. Based on the complement of the image contour $\left(C_{S}\right)$, which is obtained by subtracting the pixel value of the contour image $S$ from the maximum pixel value of $(S)$, $S_{\max }$ is as follows:

$$
C o_{S}=S_{\max }-S
$$

The MMD's coefficients are thresholded according to the $C_{S}$ image values, as follows:

$$
\hat{D}_{C, 3}^{(j)}=\left\{\begin{array}{l}
\beta \text { if } \quad \beta-\beta_{t} \leq D_{C}^{(j)} \leq \beta+\beta_{t} \\
D_{C}^{(j)} \times \nu-\left(\gamma \times C o_{S}\right) \text { if } D_{C}^{(j)}-\left(\gamma \times C o_{S}\right) \leq \beta \\
D_{C}^{(j)} \times \nu+\left(\gamma \times C o_{S}\right) \text { if } D_{C}^{(j)}+\left(\gamma \times C o_{S}\right) \geq \beta \\
\beta \quad \text { otherwise }
\end{array}\right.
$$

\section{5. $M M D$ reconstruction}

The three obtained $\hat{D}_{C, 1}^{(J)}, \hat{D}_{C, 2}^{(j)}, \hat{D}_{C, 3}^{(j)}$ are reconstructed by MMD synthesis into the enhanced images $\hat{I}_{1}, \hat{I}_{2}, \hat{I}_{3}$. It should be noted that the approximate

component $y_{1}$ is the same for the three outputs and that $\hat{D}_{C, j}^{(J)}$ are computed as follows:

$$
\begin{aligned}
& \hat{D}_{C, 1}^{(J)}=\left(y_{2 H}^{(J)}, y_{2 V}^{(J)}, y_{2 D}^{(J)}\right)_{1} \\
& \hat{D}_{C, 2}^{(J)}=\left(y_{2 H}^{(J)}, y_{2 V}^{(J)}, y_{2 D}^{(J)}\right)_{2} \\
& \hat{D}_{C, 3}^{(J)}=\left(y_{2 H}^{(J)}, y_{2 V}^{(J)}, y_{2 D}^{(J)}\right)_{3}
\end{aligned}
$$

Here, we will describe a reconstruction on an image $\hat{I}$ from $\left(y_{1}, y_{2 H}^{(J)}, y_{2 V}^{(J)}, y_{2 D}^{(J)}\right)$. Let us consider one resolution of the reconstructed signal: the nonlinear synthesis filters $r_{i j}$ represented on Fig. ??, are defined by the following equations:

$$
\begin{gathered}
r_{11}\left(y_{2 h}, y_{2 v}, y_{2 d}\right)=\frac{1}{1+\frac{h_{12}}{h_{11}} \gamma_{v}+\frac{h_{21}}{h_{11}} \gamma_{h}+\frac{h_{22}}{h_{11}} \gamma_{d}} \\
r_{12}\left(y_{2 h}, y_{2 v}, y_{2 d}\right)=\gamma_{v} r_{11}\left(y_{2 h}, y_{2 v}, y_{2 d}\right) \\
r_{21}\left(y_{2 h}, y_{2 v}, y_{2 d}\right)=\gamma_{h} r_{11}\left(y_{2 h}, y_{2 v}, y_{2 d}\right)
\end{gathered}
$$




$$
r_{22}\left(y_{2 h}, y_{2 v}, y_{2 d}\right)=\gamma_{d} r_{11}\left(y_{2 h}, y_{2 v}, y_{2 d}\right)
$$

where

$$
\begin{aligned}
& \gamma_{v}=\left\{\begin{array}{rr}
\frac{y_{2 v}}{\beta}, & y_{2 v} \leq \beta \\
\frac{1}{2-y_{2 v} / \beta} & \text { otherwise }
\end{array}\right. \\
& \gamma_{h}=\left\{\begin{array}{rr}
\frac{y_{2 h}}{\beta}, & y_{2 h} \leq \beta \\
\frac{1}{2-y_{2 h} / \beta} & \text { otherwise }
\end{array}\right. \\
& \gamma_{d}=\left\{\begin{array}{rr}
\frac{y_{2 d}}{\beta}, & y_{2 d} \leq \beta \\
\frac{1}{2-y_{2 d} / \beta} & \text { otherwise }
\end{array}\right.
\end{aligned}
$$

According to equations (??)-(??), the nonlinear response filters $r_{i j}$ are expressed as a function of the nonlinear outputs $y_{2 h}, y_{2 v}$ and $y_{2 d}$. The recon-

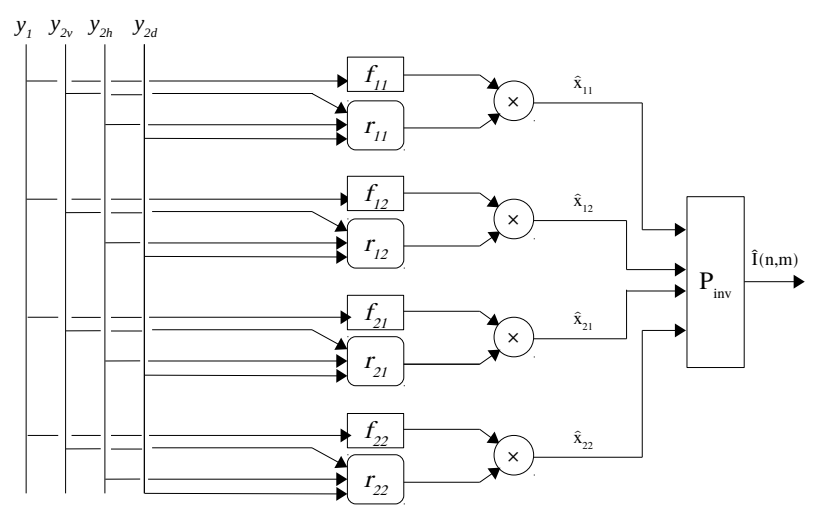

Figure 6: The 2D MMD synthesis scheme

structed polyphase components $\hat{x}_{k l}$ are expressed as follows:

$$
\begin{gathered}
\hat{x}_{11}=f_{11} \times y_{1} \times r_{11}\left(y_{2 h}, y_{2 v}, y_{2 d}\right) \\
\hat{x}_{12}=f_{12} \times y_{1} \times \gamma_{v} \times r_{11}\left(y_{2 h}, y_{2 v}, y_{2 d}\right) \\
\hat{x}_{21}=f_{21} \times y_{1} \times \gamma_{h} \times r_{11}\left(y_{2 h}, y_{2 v}, y_{2 d}\right) \\
\hat{x}_{22}=f_{22} \times y_{1} \times \gamma_{d} \times r_{11}\left(y_{2 h}, y_{2 v}, y_{2 d}\right)
\end{gathered}
$$

These equations represent the reconstructed signal as a product of a smooth component $f i j \times y_{1}$, and a component containing all the signal variations expressed as localized directional ratios $y_{2 h}, y_{2 v}, y_{2 d}$. Then, the reconstructed 
image $\hat{I}$ by MMD synthesis, is obtained by subtracting the value 1 from the result of the reconstructed polyphase components as follows:

$$
\hat{I}(n+i-1, m+j-1)=\hat{x}_{i j}(n, m) \quad i, j \in\{1,2\}
$$

where $\mathrm{n}=1, \ldots, \mathrm{N}$ and $\mathrm{m}=1, \ldots, \mathrm{M}$

For the multi-resolution synthesis, the reconstructed process is iterated to produce successive approximations based on the set $R$ (cf. equation ??). The final synthesized image is obtained at resolution $j=1$.

\section{Experimental framework}

In order to evaluate the performance of the proposed MOF-MMD, two kinds of tests are conducted: quality assessment using objective metrics and qualitative visual evaluation by medical experts. The proposed filter performance is compared with two recent and efficient speckle reduction filters, OBNLM ? and ADMSS ?.

\subsection{Image dataset}

The original clinical US images of in vivo abdominal liver were obtained from a retrospective database of the University Hospital of Angers in France. The 21 experimental images of different liver with different image size $(1080 \times$ $810,1024 \times 768$ ) were captured by SuperSonic Aixplorer and the Siemens Acuson S2000 system. The images are of granular, smooth, cirrhotic and non-cirrhotic liver. The ethical approval to use image after anonymization was obtained from the University Hospital of Angers. The images were registered in an external $\mathrm{PC}$ and processed offline.

\subsection{Evaluation metrics}

The evaluation is carried out, on the 21 ultrasound images, in terms of speckle reduction capacity and the improvement of image quality. To quantify the speckle reduction achieved by different filters, the speckle's signal-to-noise ratio (SSNR) is measured ?. For the quality evaluation metrics, three Image Quality Assessment (IQA) metrics were also chosen to assess the quality of the filtered images. As there is no genuine reference image in our cases, we consider blind objective metrics. In this paper, we use two state-of-the-art noreference metrics (NIQE ? and BIQES ?) and our previously proposed blind metric NIQE-K ?.

\subsection{1. $S S N R$}

It is measured in a fully formed region and expressed by

$$
S S N R=\frac{\mu}{\sigma}
$$

where $\mu$ is the mean intensity value and $\sigma$ is the standard deviation in the region of fully formed speckle. A higher value of the SSNR indicates less speckle. 


\subsection{2. $N I Q E$}

The NIQE ? is a perceptual metric originally proposed for evaluating the quality of a natural image. In ?? the authors conclude that the NIQE shows a good performance in medical image quality evaluation even if it is not specifically designed for this purpose. It is based on constructing a collection of features from a corpus of natural images, and fitting them to a multivariate Gaussian (MVG) model. The NIQE is both opinion- and distortion-unaware. It is expressed as the distance between constructed features and features extracted from the assessed image:

$$
N I Q E=\sqrt{\left(\left(\nu_{1}-\nu_{2}\right)^{T}\left(\frac{\sum_{1}+\sum_{2}}{2}\right)\left(\nu_{1}-\nu_{2}\right)\right)}
$$

where $\nu_{1}, \nu_{2}$ and $\sum_{1}, \sum_{2}$ are the mean vectors and covariance matrices of the reference image's MVG model and the distorted image's MVG model, respectively. It estimates the image quality only from spatial domain.

\subsubsection{BIQES}

The BIQES ? uses intrinsic features of the image. It transforms the test image into a scale-space representation, and measures the global dissimilarity with the co-occurrence histograms of the original and its scaled images (i.e., the dissimilarity between the image itself and its lower resolution versions). The first dissimilarity, called low pass error $Q_{L}$, is calculated by comparing low pass versions across scales with the original image. The second dissimilarity, called high pass error $Q_{H}$, is computed from the variance and gradient histograms, weighted by the contrast sensitivity function in order to make it perceptually effective. These two dissimilarities are combined together to derive the final quality score:

$$
B I Q E S=\frac{k}{\sigma} \overline{Q_{L}}+\sigma \overline{Q_{H}}
$$

where $k$ and $\sigma$ are the kurtosis and the standard deviation of the log amplitude of the image's Fourier spectra, respectively. ' denotes the mean operation of $Q_{L}$ and $Q_{H}$ the calculated low pass error and high pass one.

\subsection{4. $N I Q E_{K}$}

The $N I Q E_{K}$ ? combines some low level features of the image from BIQES with the NIQE to assess the quality of processed medical images.

$$
N I Q E_{K}=N I Q E \times \frac{k}{\sigma}
$$

A lower value of the metrics (NIQE, BIQES and NIQE-K) indicates a better quality of the image. 


\subsection{Subjective experiment}

\subsubsection{Human observer}

For our study, three radiologists with different years of experience (3 years, 7 years and more than 10 years experience) were asked to score the perceived quality of the filtered images, based on four criteria: the image contrast, the ability to diagnose, the texture conspicuity and the edge sharpness. The radiologists are from the affiliated Hospital of Nanjing Medical University in China, thus they haven't seen the test images before. The assessment was conducted in an environment similar to the one in which radiologists practice daily.

The number of radiologists in this study is comply with the European guidelines on quality criteria for diagnostic radiographic images?, which recommends to involve two observers at least to assess each image with the criteria independently.

\subsubsection{Subjective test methodology}

The subjective testing methodology impacts both the accuracy and the reliability of the collected perceived quality scores. For the subjective quality assessment of the medical images, various testing methods have been used through the literature. The most used methodologies are:

- The Double-Stimulus Continuous Quality Scale (DSCQS) where the observer is presented two stimuli side by side, typically the reference and the impaired one ?

- The Subjective AssessMent for VIdeo Quality (SAMVIQ) where the observer freely views and scores a collection of test stimuli associated with an explicitly identified reference stimulus?

- The Absolute Categorical Rating (ACR) where the observer is presented with a stimulus, then the display is set to a constant gray background and the observer is immediately requested to provide an opinion score of the viewed stimulus?

According to ? Simple Stimulus (SS) protocols perform better than Double Stimulus (DS) ones in terms of subject fatigue and avoidance of mistakes due to accidentally reversing scores in DS. Additionally, SAMVIQ scores with greater accuracy compared to ACR for the same number of observers (on average $30 \%$ fewer observers were required) and is more reliable for the perceived quality scores of the collected data ?. For the above cited reasons, we choose the SAMVIQ method in this test since it provides more accurate and reliable perceived quality scores on collected data.

In the SAMVIQ protocol the images are presented to the observer (assessor) such that he can evaluate all processed versions of the image as well as against the reference. SAMVIQ offers the possibility to visualize each image several times and re-evaluate a previously scored image. The test according to SAMVIQ is conducted task by task. Each task includes an explicit reference, a hidden reference and all processed versions: MOF-MMD OUTPUT1-2-3, ADMSS and 
OBNLM. The explicit and the hidden are used as quality anchors that stabilize the results and improve the consistency of the scores. The number of reference images is limited to 12 as the subjective test, particularly with the SAMVIQ protocol, is intrinsically time-consuming.

The stimuli are randomized in order to prevent the assessors from attempting to vote in an identical way according to an established order ?. A training session is conducted by a test supervisor in order to make the assessor familiar with image artifacts and the user interface ?. Fig. ?? shows the graphical user interface (GUI) of the experiment. The observer is asked to score each stimulus using a continuous rating scale from 0 to 100. The rating scale is categorized according to the adjectives Low, Medium and High. The observer is allowed to view a stimulus multiple times in a task and change the score, which lengthens the duration of the test session.

In the purpose avoiding observer fatigue, the test is divided into 2 sessions. In each session, 2 criterion are evaluated by asking the following questions to assessors:

- Session 1

- Diagnosis: Please score how well the enhanced image helps the diagnosis.

- Contrast: Please score the contrast improvement of the enhanced image (difference between the tissue and the background).

- Session 2

- Texture conspicuity: Please score the general clarity of the texture.

- Edge sharpness: Please score the visibility of the tissue border

Representative images for each criterion with associated score are shown in Fig. ?? and ??.

The image ?? has less difference in brightness between the light and dark parts (i.e., contrast) than image ??. On the other hands some edges are depicted in Fig. ??, and are more visible inside the blues circles than inside the reds ones.

The images ?? and ?? show more clearly the difference in texture enhancement. The red and blue circles represent two areas where the grain pattern is smooth and raised, respectively.

\subsubsection{Subjective scores}

The obtained scores were averaged across despeckling methods to yield a Mean Opinion Scores (MOS) for each subject.

$$
M O S_{j}=\frac{1}{n b} \sum_{i=1}^{n b} r_{i j}
$$

Where $n b$ is the total number of the image, $r_{i j}$ represents the raw score of the i-th image given by the j-th subject. 


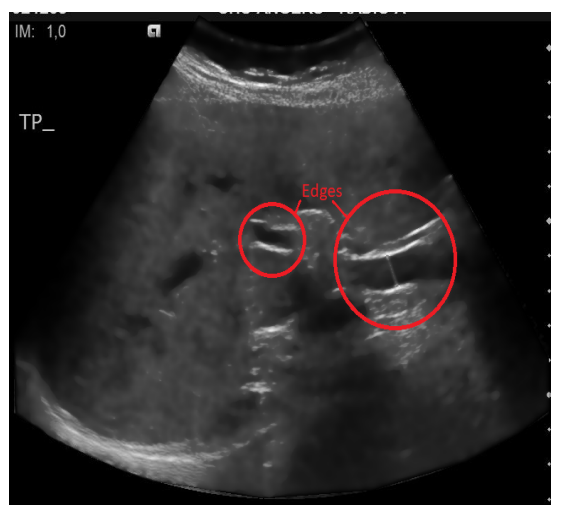

(a) Contrast score $=16.66$ Edge score $=23.33$

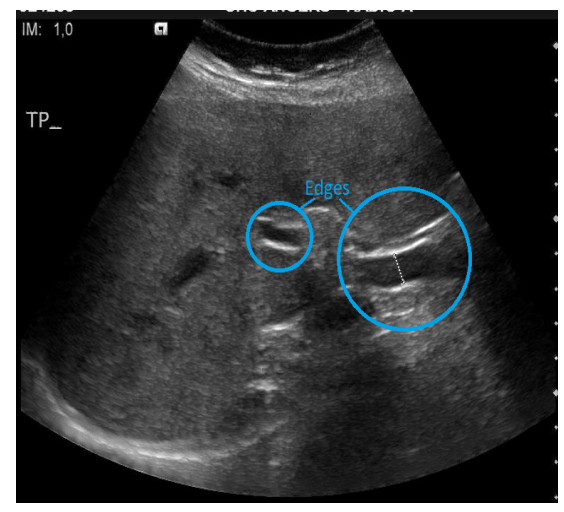

(b) Contrast score $=80$ Edge score $=80$

Figure 7: Illustration of evaluated contrast and edge criterion with associate subjective score.

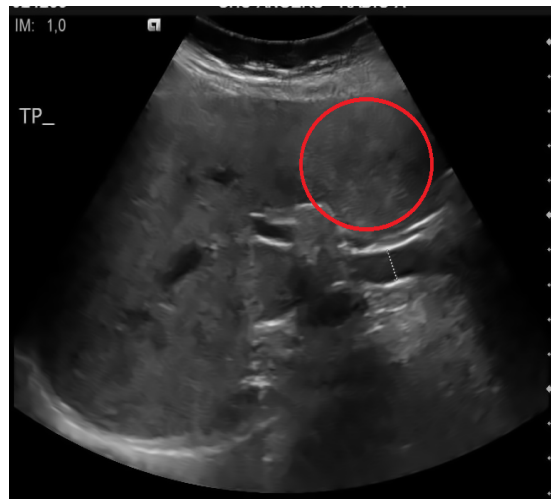

(a) Texture score $=16.66$

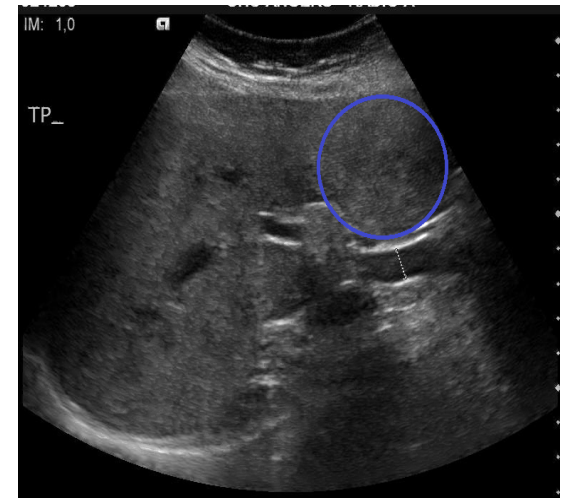

(b) Texture score $=86.66$

Figure 8: Illustration of evaluated texture criterion with associated subjective score 


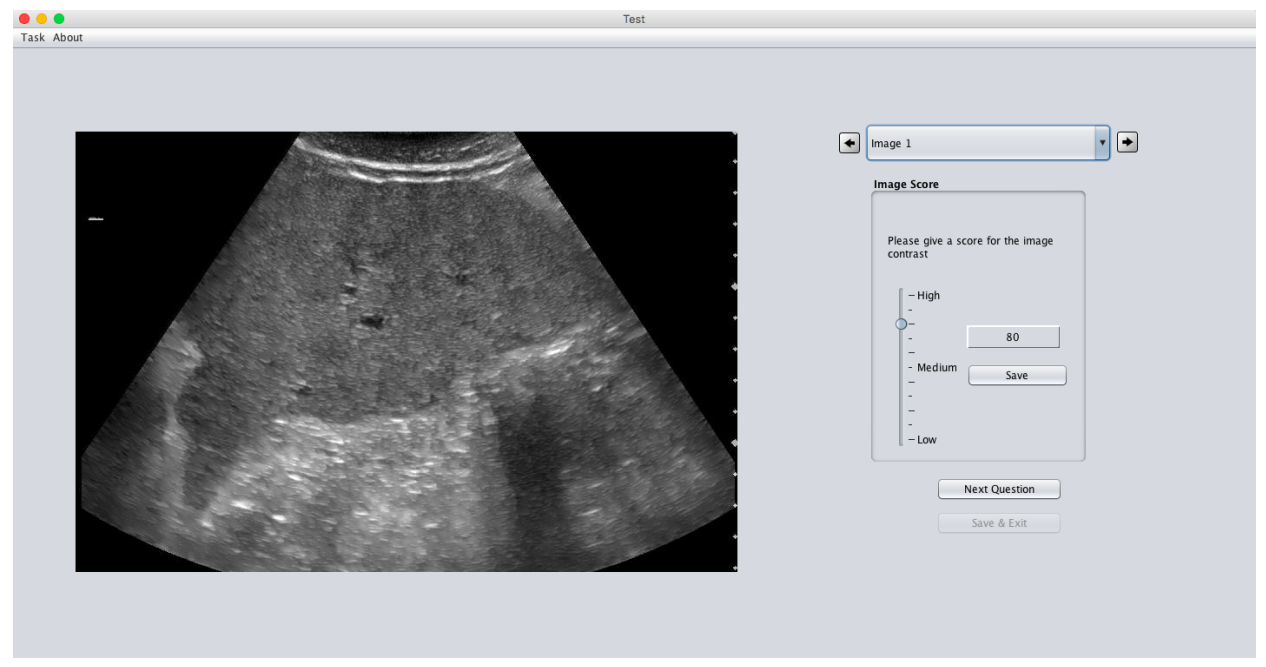

Figure 9: Graphical User Interface

\section{Results and discussions}

\subsection{Objective evaluation results}

For objective comparison, the SSNR and the three quality metrics are calculated and depicted with a box plot in Fig. ??. Considering the speckle reduction capacity shown by the SSNR in Fig. ??, the proposed method as well as the OBNLM and ADMSS reduce the speckle while the OBNLM filter has a slightly better performance. However, the OBNLM filter offers a bad image quality due to the over-smoothing effect, cf. Fig. ??and ??.

Regarding the three outputs of the proposed method, the NIQE, NIQE-K and BIQES indicate that they render a higher quality compared to the OBNLM and the ADMSS. Moreover, the standard deviations of the three outputs are lower than those of the ADMSS and OBNLM, which indicates a lower dispersion and a higher uniformity of the processed images. The MOF-MMD substantially reduces the speckle while improving the quality of the image. It provides three outputs that are useful for the diagnosis of general aspect, echo pattern and outer border.

\subsection{Subjective evaluation results}

Table ?? shows the MOSs given by radiologists for five sets of processed images shown in Fig. ??. For the four considered criteria (contrast, texture conspicuity, edge sharpness and diagnostic) the three medical experts mostly scored the proposed method as better. The OBNLM method has relatively lower scores due to the artificial appearance and the oversmoothing texture which is unnatural for the radiologists. Considering the texture conspicuity, the three radiologists find Output1 more noticeable, meaning that the features-like segmentation based on mathematical morphology is also valuable for texture 


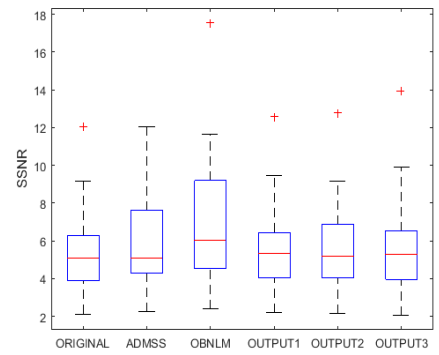

(a) SSNR

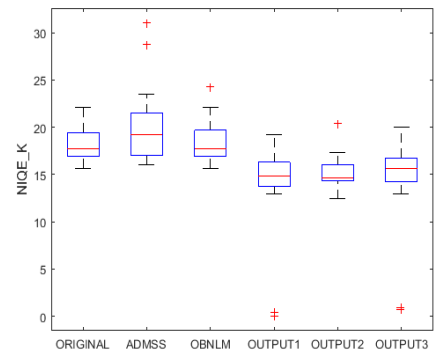

(c) NIQE-K

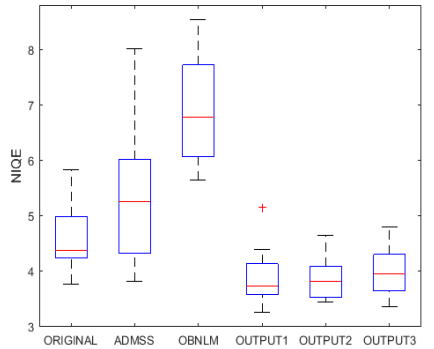

(b) NIQE

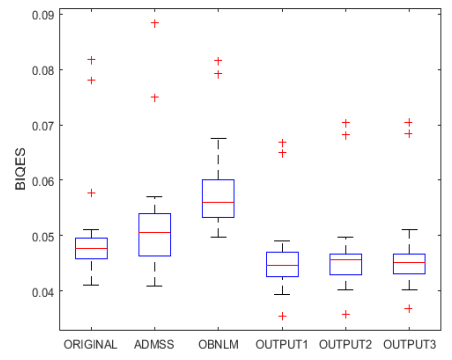

(d) BIQES

Figure 10: Box plots of SSNR and quality assessment metrics of US images and their filtered versions: ADMSS, OBNLM, proposed OUTPUT1-3. (A higher SSNR indicates less speckle. The lower values of metrics means higher performances) 
enhancement. For contrast enhancement, according to three radiologists, the MOF-MMD Output1 improves contrast. Considering the edge sharpness and the diagnostic parameters, radiologist 1 indicates a better diagnostic and sharpness of the edges on output3, radiologist 2 scores output 1 as that which best enhances the edges and radiologist 3 finds output 2 as the best one. This result suggests that the edge sharpness coincides with the diagnostic facility and that the years of experience of the radiologist may be an important factor for making a diagnosis. The great interest of the proposed method is that it provides radiologists with the possibility of choosing an output as they wish according to their experiences and needs in different circumstances.

Another observation from the subjective scoring is the difference between the initial hypothesis and the results. In fact, the multi-output filter supposes initially output 1 for edge sharpness, output 2 for texture enhancement and output 3 for general improvement of the image quality supposed to facilitate the diagnostic task. This experiment shows that output 1 , considering the scores of radiologists and all criteria (contrast, texture conspicuity, edges sharpness and diagnostic), is generally the most adequate as it is ranked best most often, all parameters combined. Finally, this latter finding about output 1 suggests that the features-like segmentation by morphological operators is a very interesting step for enhancing, and could help the application of image processing techniques in US images.

Table 1: Subjective mean scores of speckle filtering methods

\begin{tabular}{|c|c|c|c|c|c|c|c|c|c|c|c|c|}
\hline & \multicolumn{4}{|c|}{ Radiol1 } & \multicolumn{4}{|c|}{ Radiol2 } & \multicolumn{4}{|c|}{ Radiol3 } \\
\hline & Contrast & Texture & Edges & Diagn. & Contrast & Texture & Edges & Diagn. & Contrast & Texture & Edges & Diagn. \\
\hline ADMSS & 43.33 & 50 & 43.33 & 39.16 & 46.66 & 52.5 & 50.83 & 48.33 & 55.83 & 56.66 & 52.5 & 51.66 \\
\hline OBNLM & 45.45 & 34.54 & 32.72 & 37.27 & 49.09 & 40.83 & 47.27 & 41.81 & 33.63 & 38.18 & 30.91 & 26.36 \\
\hline $\begin{array}{l}\text { Proposed } \\
\text { Output1 }\end{array}$ & 50 & 76.66 & 79.16 & 77.5 & 55.83 & 66.66 & 79.16 & 64.16 & 76.66 & 78.33 & 69.16 & 69.16 \\
\hline $\begin{array}{l}\text { Proposed } \\
\text { Output2 }\end{array}$ & 39.16 & 75.83 & 79.16 & 80.83 & 55 & 65 & 69.16 & 55.83 & 75.45 & 70 & 72.73 & 79.09 \\
\hline $\begin{array}{l}\text { Proposed } \\
\text { Output3 }\end{array}$ & 42.5 & 75.83 & 81.66 & 81.66 & 53.33 & 66.66 & 76.66 & 56.66 & 74.16 & 66.66 & 70.83 & 75.83 \\
\hline
\end{tabular}

\subsubsection{Statistical analysis}

The collected scores are further analyzed statistically with ANOVA (Analysis of Variance) using MATLAB's ANOVA function. For each test, the perceived quality is selected as the dependent variable. The image content's, the despeckleing algorithms and the observers are selected as independent variable. The results are summarized in Table ??, and show that there is no significant difference between observers ( $\mathrm{P}$-value $>0.05)$ in scoring the image quality. Furthermore, the image content and despeckling method both have a significant effect on perceived quality $(\mathrm{P}$-value $<0.05)$. The impact of image content is probably due to the fact that the original images are different in terms of parenchymal tissue echogenicity (i.e., the tissue ability to bounce an echo). A statistical T-test is further performed for pairwise comparisons with hypothesis testing between the despeckling algorithms. The results are summarized in Table ??, 


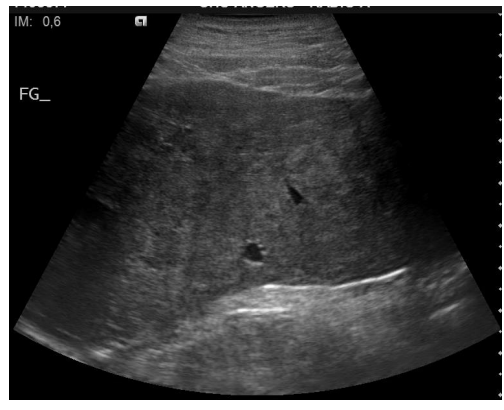

(a) Original

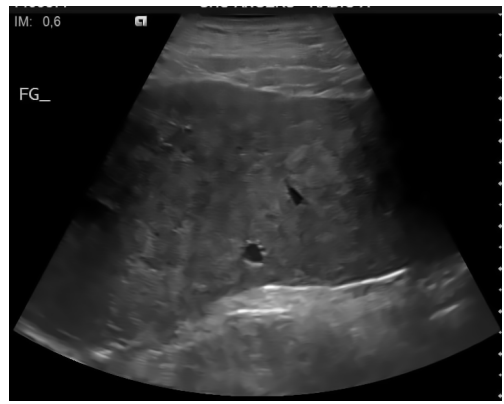

(c) OBNLM

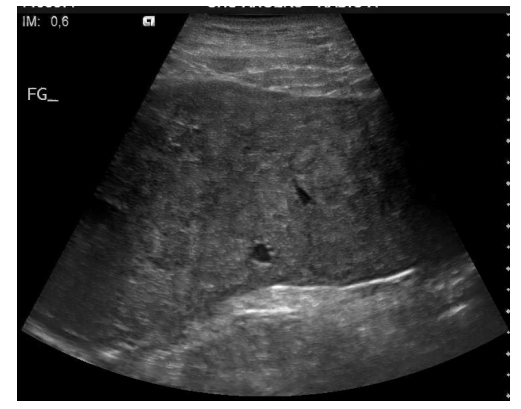

(e) Proposed Output2: Texture

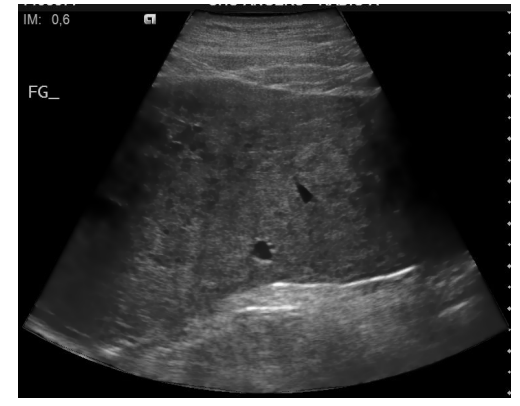

(b) ADMSS

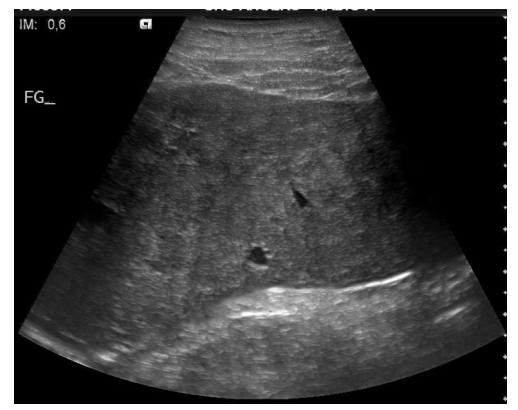

(d) Proposed Output1: Edges

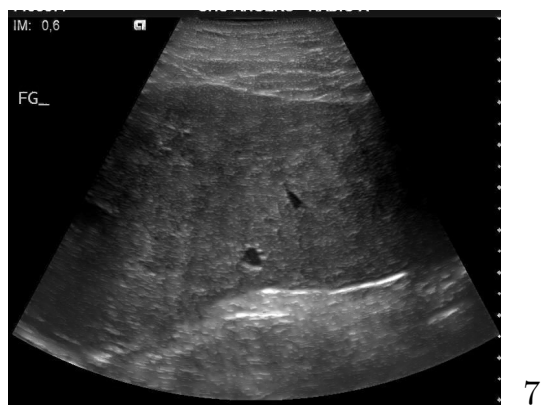

(f) Proposed Output3: Global

Figure 11: Subjective comparison of speckle reduction of liver US image 
and indicate that the perceived quality is significantly better for the proposed method (OUTPUT1, OUTPUT2 and OUTPUT 3). For the pairwise comparisons: OUTPUT1 vs OUTPUT2, OUTPUT2 vs OUTPUT3 and OUTPUT1 vs OUTPUT3 the difference is not statistically significant for each case.

Table 2: Results of ANOVA to evaluate the effect of the observer, content and despeckling on the perceived quality

\begin{tabular}{|c||c|c|c|}
\hline Factor & $\mathrm{dF}$ & $\mathrm{F}$ & $\mathrm{P}$-value \\
\hline Observer & 2 & 2.25 & 0.106 \\
\hline Content & 11 & 2.27 & 0.009 \\
\hline Despeckling algorithms & 4 & 68.04 & $<0.001$ \\
\hline
\end{tabular}

\subsection{2. analysis}

In this section we briefly analyze the correlation between objective metrics and the collected perceived scores. Being able to predict the perceived image quality would help to shorten the times of the subjective tests. The two wellknown correlation coefficients: PLCC (Pearson Linear Correlation Coefficient) and SROCC (Spearman Rank Order Correlation Coefficient) are used as correlation metrics. PLCC requires constructing nonlinear mapping between objective metrics and subjective scores using Logistic regression?.

Table 3: T-Test of statistical significance for pairwise comparisons of despeckling method. Three symbols: "1" means that the method for the row is significantly better than the method for the column, "-1" means that it is significantly worse, and " 0 " means that it is statistically indistinguishable

\begin{tabular}{|c|c|c|c|c|c|}
\hline & ADMSS & OBNLM & OUTPUT1 & OUTPUT2 & OUTPUT3 \\
\hline ADMSS & 0 & -1 & 1 & 1 & 1 \\
\hline OBNLM & 1 & 0 & 1 & 1 & 1 \\
\hline OUTPUT1 & -1 & -1 & 0 & 0 & 0 \\
\hline OUTPUT2 & -1 & -1 & 0 & 0 & 0 \\
\hline OUTPUT3 & -1 & -1 & 0 & 0 & 0 \\
\hline
\end{tabular}

$$
M_{P}=\beta_{1}\left(\frac{1}{2}-\frac{1}{1+\exp \left(\beta_{2}\left(M-\beta_{3}\right)\right)}\right)+\beta_{4} M+\beta_{5}
$$

where $M$ and $M_{p}$ are the original and the fitted objective NR-IQA scores respectively. $\beta_{1}, \beta_{2}, \beta_{3}, \beta_{4}, \beta_{5}$ are the regression parameters of the logistic function. The results of correlation between the three metrics and the perceived quality of contrast, diagnostic, texture and edges, are summarized in Table ??. One can conclude that the NIQE metric is reliable to predict the perceived quality by radiologists for the diagnostic task. Moreover, the characterization of the lesion is well predicted by the $N I Q E$ as edge and texture perception by radiologists is highly correlated with the NIQE. However, the contrast perceived by the radiologists is far from the one assessed by the three objective metrics used. 
Table 4: PLCC after nonlinear regression and SROCC between MOS and NR-IQ metrics

\begin{tabular}{|c|c|c|c|c|c|c|}
\cline { 2 - 7 } \multicolumn{1}{c|}{} & \multicolumn{3}{c|}{ PLCC } & \multicolumn{3}{c|}{ SROCC } \\
\hline Criterion & NIQE & NIQEK & BIQES & NIQE & NIQEK & BIQES \\
\hline Contrast & 0.34 & 0.30 & 0.23 & 0.25 & 0.10 & 0.37 \\
\hline Diagnostic & 0.82 & 0.50 & 0.33 & 0.56 & 0.38 & 0.30 \\
\hline Texture & 0.79 & 0.43 & 0.65 & 0.46 & 0.34 & 0.56 \\
\hline Edges & 0.88 & 0.61 & 0.72 & 0.61 & 0.54 & 0.60 \\
\hline
\end{tabular}

\subsubsection{Limitation of the proposed method}

The selection of optimal threshold is the main limitation of the thresholding filter. Hence, the thresholding may be improved particularly to adapt it to other imaging modalities. The statistical analysis suggests that the content of the image has a significant effect on the perceived quality. It would be beneficial to study the impact of the image content: healthy versus pathological, granular versus smooth on the diagnostic performance. Finally, one can note that the ultrasound examination is used in real time for a wide variety of clinical tasks. So, it would be of great interest to embed a speckle filter into ultrasound imaging systems. Also, further improved results can be expected by incorporating some feature learning based strategies into the proposed methods, such as dictionary learning or deep convolution learning ???.

\section{Conclusion}

Speckle reduction is useful preprocessing step for US imaging. In this paper a fully automatic multi-output filter based on multiplicative multiresoution decomposition (MOF-MMD) is proposed to improve the ability of interpreting the ultrasound image for the medical user. The MOF-MMD permits enhancement of the image in multiple ways, according to the structure that needs to be viewed. We employ both the specificity of the multi-scale decomposition (MMD) and the advantage of mathematical morphology to delimit the undesirable structures. To evaluate the performance of the proposed method, the SSNR and three blind quality metrics are used to quantify the speckle reduction and quality improvement of the US image. Moreover, a subjective evaluation is carried out according to the recommendation with the SAMVIQ protocol. The visual evaluation shows that the proposed method with its various outputs is more or less valuable according to the different years of experience of the radiologists. The NIQE metric has the best correlation with the subjective scores in our test, this alternatively allows comparison of the medical ultrasound, however its performance is still not satisfied and can be further improved in future works. In our future work we propose to study the impact of the image content on the perceived quality on the one hand and to include this depeckeling method in a lossy multiresolution compression on the other hand.

\section{References}


Achim A, Bezerianos A and Tsakalides P 2001 Novel Bayesian multiscale method for speckle removal in medical ultrasound images IEEE Trans. Med. Imaging 20 772-83

Aja-Fernández S and López C A 2006 On the estimation of the coefficient of variation for anisotropic diffusion speckle filtering IEEE Trans. Image Process. 15 2694-701

Buades A, Coll B and Morel J 2005 A review of image denoising algorithms, with a new one Multiscale Model. Simul. 4 490-530

Burckhardt C B 1978 Speckle in ultrasound B-mode scans IEEE Trans. Sonics Ultrason. 25 1-6

Chen Y, Chen W, Yin X, Ye X, Bao X, Luo L, Feng Q, Li Y and Yu X 2011 Improving low-dose abdominal CT images by weighted intensity averaging over large-scale neighborhoods Eur. J. Radiol. 80 e42-9

Chen Y, Ma J, Feng Q, Luo L, Shi P and Chen W 2008 Nonlocal prior Bayesian tomographic reconstruction J. Math. Imaging Vis. 30 133-46

Chen Y, Yang Z, Hu Y, Yang G, Zhu Y, Li Y, Luo L, Chen W and Toumoulin C 2012 Thoracic low-dose CT image processing using an artifact suppressed large-scale nonlocal means Phys. Med. Biol. 57 266788

Chen Y, Zhang Y, Shu H, Yang J, Luo L, Coatrieux J L and Feng Q 2018 Structure-adaptive fuzzy estimation for random-valued impulse noise suppression IEEE Trans. Circuits Syst. Video Technol. 28 414-27

Coupé $\mathrm{P}$, Hellier P, Kervrann C and Barillot C 2009 Nonlocal means-based speckle filtering for ultrasound images IEEE Trans. Image Process. 18 2221-9

Déforges O, Normand N and Babel M 2013 Fast recursive grayscale morphology operators: from the algorithm to the pipeline architecture J. Real-Time Image Process. 8 143-52

Donoho D L 1995 De-noising by soft-thresholding IEEE Trans. Inf. Theory 41 613-27

European Commission 1996 European Guidelines on Quality Criteria for Diagnostic Radiographic Images (Luxembourg: Office for Official Publications of the European Communities)

Frost V S, Stiles J A, Shanmugan K S and Holtzman J C 1982 A model for radar images and its application to adaptive digital filtering of multiplicative noise IEEE Trans. Pattern Anal. Mach. Intell. 4 157-66 
Gai S, Zhang B, Yang C and Yu L 2018 Speckle noise reduction in medical ultrasound image using monogenic wavelet and Laplace mixture distribution Digit. Signal Process.: Rev. J. 72 192-207

Gerstenmaier J F and Gibson R N 2014 Ultrasound in chronic liver disease Insights Imaging 5 441-55

Imbault M, Faccinetto A, Osmanski B F, Tissier A, Deffieux T, Gennisson J L, Vilgrain V and Tanter M 2017 Robust sound speed estimation for ultrasound-based hepatic steatosis assessment Phys. Med. Biol. 623582

Kang J, Lee J Y and Yoo Y 2016 A new feature-enhanced speckle reduction method based on multiscale analysis for ultrasound B-mode imaging IEEE Trans. Biomed. Eng. 63 1178-91

Kozamernik F and Sunna E W P 2005 Subjective quality of internet video codecs: evaluation using SAMVIQ EBU Technical Review (Switzerland: European Broadcasting Union)

Krissian K, Westin C, Kikinis R and Vosburgh K G 2007 Oriented speckle reducing anisotropic diffusion IEEE Trans. Image Process. 16 1412-24

Kuan D T, Sawchuk A A, Strand T C and Chavel P 1985 Adaptive noise smoothing filter for images with signal-dependent noise IEEE Trans. Pattern Anal. Mach. Intell. 7 165-77

Kumcu A, Bombeke K, Platisa L, Jovanov L, Looy J V and Philips W 2017 Performance of four subjective video quality assessment protocols and impact of different rating preprocessing and analysis methods IEEE J. Sel. Top. Signal Process. 11 48-63

Lee J 1980 Digital image enhancement and noise filtering by use of local statistics IEEE Trans. Pattern Anal. Mach. Intell. 2 165-8

Lee J 1981 Speckle analysis and smoothing of synthetic aperture radar images Comput. Gr. Image Process. 17 24-32

Liu J, Hu Y, Yang J, Chen Y, Shu H, Luo L, Feng Q, Gui Z and Coatrieux G 2017a 3D feature constrained reconstruction for low dose CT imaging IEEE Trans. Circuits Syst. Video Technol. 28 1232-47

Liu J et al 2017b Discriminative feature representation to improve projection data inconsistency for low dose CT imaging IEEE Trans. Med. Imaging 36 2499-509

Locatelli A, Piccoli M G, Vergani P, Mariani E, Ghidini A, Mariani S and Pezzullo J C 2000 Critical appraisal of the use of nuchal fold thickness measurements for the prediction of down syndrome Am. J. Obstetrics Gynecol. 182 192-7 
Loizou C P, Murray V, Pattichis M S, Pantziaris M, Nicolaides A and Pattichis C S 2014 Despeckle filtering for multiscale amplitude-modulation frequency-modulation (AM-FM) texture analysis of ultrasound images of the intima-media complex Int. J. Biomed. Imaging 2014

Loizou C P, Pattichis C S, Christodoulou C I, Istepanian R S, Pantziaris M and Nicolaides A 2005 Comparative evaluation of despeckle filtering in ultrasound imaging of the carotid artery IEEE Trans. Ultrason. Ferroelectr. Freq. Control 52 1653-69

Mittal A, Soundararajan R and Bovik A C 2013 Making a 'completely blind' image quality analyzer IEEE Signal Process. Lett. 20 209-12

Outtas M, Serir A and Kerouh F 2014 Speckle noise reduction in ultrasound image based on a multiplicative multiresolution decomposition (MMD) The 8th Edition of Int. Symp. on Signal, Image, Video and Communications (Marrakech, Morocco, 2014)

Outtas M, Zhang L, Deforges O, Hammidouche W, Serir A and Cavaro-Menard C 2016 A study on the usability of opinion-unaware no-reference natural image quality metrics in the context of medical images Int. Symp. on Signal, Image, Video and Communications (Tunis, Tunisia, 21-23 November 2016)

Outtas M, Zhang L, Deforges O, Serir A and Hamidouche W 2017 Multi-output speckle reduction filter for ultrasound medical images based on multiplicative multiresolution decomposition IEEE Int. Conf. on Image Processing (Beijing, China, 2017)

Perona P and Malik J 1990 Scale-space and edge detection using anisotropic diffusion IEEE Trans. Pattern Anal. Mach. Intell. 12 629-39

Pizurica A, Philips W, Lemahieu I and Acheroy M 2003 A versatile wavelet domain noise filtration technique for medical imaging IEEE Trans. Med. Imaging 22 323-31

Ramos-Llorden G, Vegas-Sanchez-Ferrero G, Martin-Fernandez M, Alberola-Lopez C and AjaFernández S 2014 Anisotropic diffusion filter with memory based on speckle statistics for ultrasound images IEEE Trans. Image Process. 24 345-58

Ramponi G, D'Alvise R and Moloney C 1999 Automatic estimation of the noise variance in SAR images for use in speckle filtering Proc. of the IEEE-EURASIP Workshop on Nonlinear Signal and Image Processing (Antalya, Turkey, 20-23 June 1999)

Razaak M and Martini M G 2016 Cuqi: cardiac ultrasound video quality index J. Med. Imaging 3 011011

Ritenour E R, Nelson T R and Raff U 1984 Applications of the median filter to digital radiographic images IEEE Int. Conf. on Acoustics, Speech, and Signal Processing (San Diego, CA, 19-21 March 1984) 
Rouse D M, Pépion R, Callet P L and Hemami S S 2010 Tradeoffs in subjective testing methods for image and video quality assessment Human Vision and Electronic Imaging XV, part of the IS\&T-SPIE Electronic Imaging Symp., Proc. (San Jose, CA, 18-21 January 2010)

Sa-ing V, Vorasayan P, Suwanwela N C, Auethavekiat S and Chinrungrueng C 2018 Multiscale adaptive regularisation Savitzky-Golay method for speckle noise reduction in ultrasound images IET Image Process. 12 105-12

Saha A and Wu Q M J 2015 Utilizing image scales towards totally training free blind image quality assessment IEEE Trans. Image Process. 24 1879-92

Serir A and Belouchrani A 2004 Multiplicative multiresolution decomposition for 2D signals: application to speckle reduction in SAR images Int. Conf. on Image Processing vol 1

Serir A, Beghdadi A and Kerouh F 2013 No-reference blur image quality measure based on multiplicative multiresolution decomposition J. Vis. Commun. Image Represent. 24 911-25

Sheikh H R, Bovik A C and Cormack L K 2005 No-reference quality assessment using natural scene statistics: JPEG2000 IEEE Trans. Image Process. 14 1918-27

Sonka M, Hlavac V and Boyle R 2007 Image Processing, Analysis, and Machine Vision (New York: Springer) (Thomson-Engineering)

Sudeep P, Palanisamy P, Rajan J, Baradaran H, Saba L, Gupta A and Suri J S 2016 Speckle reduction in medical ultrasound images using an unbiased non-local means method Biomed. Signal Process.

Control 28 1-8

Vimala C and Aruna Priya P 2018 Double density dual tree discrete wavelet transform implementation for degraded image enhancement J. Phys.: Conf. Ser. 1000012120

Wagner R F, Smith S W, Sandrik J M and Lopez H 1983 Statistics of speckle in ultrasound B-scans IEEE Trans. Sonics Ultrason. 30 156-63

Wu S, Zhu Q and Xie Y 2013 Evaluation of various speckle reduction filters on medical ultrasound images 35th Annual Int. Conf. of the IEEE Engineering in Medicine and Biology Society

Yang W et al 2017 Improving low-dose CT image using residual convolutional network IEEE Access 5 24698-705

Yu Y and Acton S T 2002 Speckle reducing anisotropic diffusion IEEE Trans. Image Process. 11 126070 
Zhang F, Yoo Y M, Koh L M and Kim Y 2007 Nonlinear diffusion in laplacian pyramid domain for ultrasonic speckle reduction IEEE Trans. Med. Imaging 26 200-11

Zhang J, Wang C and Cheng Y 2015 Comparison of despeckle filters for breast ultrasound images Circuits Syst. Signal Process. 34 185-208 